\title{
Single spin resonance driven by electric modulation of the $g$-factor anisotropy
}

\author{
A. Ferrón $\odot,{ }^{1, *}$ S. A. Rodríguez $\odot,{ }^{1}$ S. S. Gómez, ${ }^{1}$ J. L. Lado, ${ }^{2}$ and J. Fernández-Rossier ${ }^{3, \dagger}$ \\ ${ }^{1}$ Instituto de Modelado e Innovación Tecnológica (CONICET-UNNE) and Facultad de Ciencias Exactas, Naturales y Agrimensura, \\ Universidad Nacional del Nordeste, Avenida Libertad 5400, W3404AAS Corrientes, Argentina \\ ${ }^{2}$ Department of Applied Physics, Aalto University, Espoo, Finland \\ ${ }^{3}$ QuantaLab, International Iberian Nanotechnology Laboratory (INL), Avenida Mestre José Veiga, 4715-330 Braga, Portugal
}

(Received 20 September 2019; revised manuscript received 12 November 2019; published 18 December 2019)

\begin{abstract}
We address the problem of electronic and nuclear spin resonance of an individual atom on a surface driven by a scanning tunneling microscope. Several mechanisms have been proposed so far, some of them based on the modulation of exchange and crystal field associated with a piezoelectric displacement of the adatom driven by the radio frequency (RF) tip electric field. Here we consider another mechanism, where the piezoelectric displacement modulates the $g$-factor anisotropy, leading both to electronic and nuclear spin flip transitions. We discuss thoroughly the cases of hydrogenated $\mathrm{Ti}(S=1 / 2)$ and $\mathrm{Fe}(S=2)$ on $\mathrm{MgO}$, relevant for recent experiments. We model the system using two approaches. First, an analytical model that includes crystal field, spin orbit coupling, and hyperfine interactions. Second, we carry out density-functional-based calculations. We find that the modulation of the anisotropy of the $g$ tensor due to the piezoelectric displacement of the atom is an additional mechanism for scanning tunneling microscopy (STM)-based single spin resonance that would be effective in $S=1 / 2$ adatoms with large spin orbit coupling. In the case of hydrogenated $\mathrm{Ti}$ on $\mathrm{MgO}$, we predict a modulation spin resonance frequency driven by the DC electric field of the tip.
\end{abstract}

DOI: 10.1103/PhysRevResearch.1.033185

\section{INTRODUCTION}

The quest of single spin electron paramagnetic resonance (EPR) driven with a scanning tunneling microscope (STM) has been pursued for many years [1,2]. The first report of STM-EPR of individual adatoms on a surface of $\mathrm{MgO}(100) / \mathrm{Ag}$ [3] has been followed by several dramatic breakthroughs in the study of spin physics of individual magnetic atoms [4-13]. This technique permits one to carry out absolute measurements of the magnetic moment of individual atoms $[4,5]$. The spectral resolution achieved so far, down to a few $\mathrm{MHz}$, has made it possible to resolve the hyperfine structures of $\mathrm{Fe}, \mathrm{Ti}$, and $\mathrm{Cu}$ atoms [8,10]. In the case of $\mathrm{Cu}$ adatoms, the electrical driving of nuclear spin flip transitions that preserve the electronic spin has been demonstrated as well [10]. Thus, STM-EPR permits one to drive the electronic and nuclear spins of individual atoms on surfaces, as well as artificially created structures, such as dimers [6,9]. Importantly, the STM-EPR technique is being now implemented in several different laboratories, at higher temperatures [14] and higher driving frequencies [15].

An important question in the STM-EPR context [3-12], and also for experiments reporting electric control of individ-

\footnotetext{
*Corresponding author: aferron@ gmail.com

†n leave from Departamento de Fisica Aplicada, Universidad de Alicante, 03690, Spain.

Published by the American Physical Society under the terms of the Creative Commons Attribution 4.0 International license. Further distribution of this work must maintain attribution to the author(s) and the published article's title, journal citation, and DOI.
}

ual nuclear spin in single molecule transport [16,17], is the understanding of how electric fields couple both to electronic and nuclear spin degrees of freedom. This question has also been addressed in other systems. The idea of electric dipole spin resonance was proposed in 1960 by Rashba [18]. Electrical control of spin qubits has been reported in semiconductor nanostructures, based both on modulation of the $g$ factor [19] and on inhomogeneous magnetic fields [20,21]. Electric fields have been used to drive spin resonance of itinerant electrons in $\mathrm{InSb}$ [22] and localized magnetic dopants in $\mathrm{ZnO}$ [23].

In the seminal paper of Baumann et al. [3], where a STMEPR experiment was carried out with $\mathrm{Fe}$ atoms on an $\mathrm{MgO}$ surface, a mechanism was proposed to account for the coupling of the STM voltage to the electronic spin that depended on the specific details of the microscopic Hamiltonian of that system. The mechanism is based on the assumption that the radio frequency $(\mathrm{RF})$ field induces a vertical piezoelectric displacement of the adatom, $\delta z \propto e V_{\mathrm{RF}}$, that in turns modifies the crystal field Hamiltonian of the $d$ orbitals of Fe. This modulation, together with spin orbit coupling and a strong in-plane Zeeman field, would lead to spin transitions between the two lowest energy states of the $S=2, S_{z}= \pm 2$ of Fe, a non-Kramers doublet integer spin system [24].

Other mechanisms have been proposed to account for the driving of the surface spin by the tip bias voltage [2,25-28]. For instance, in Ref. [26] we proposed a mechanism based on the modulation of the exchange interaction between the magnetic tip and the magnetic adatom that originates also from the piezoelectric distortion of the adatom.

Here we propose another complementary mechanism, that can coexist with the others, based on the electric modulation of the $g$ tensor associated with the piezoelectric distortion of 
the adatom. As in the case of the crystal field [3] and exchange $[11,26]$ mechanisms, we also assume that the magnetic adatom undergoes a piezoelectric displacement. In turn, this modulation changes the crystal field parameters that control the anisotropy of the electronic spin interactions that leads to an anisotropic $g$ factor and to a renormalization of the hyperfine coupling. As we show below, these modulations lead both to electronic and nuclear spin flip transitions.

The rest of this paper is organized as follows. In Sec. II, we present a general argument to show that an anisotropic timedependent modulation of the $g$ tensor of a $S=1 / 2$ system leads to electronic spin transitions. In Sec. III, we briefly present a single-particle Hamiltonian for a $d^{1}$ adatom with $C_{4}$ symmetry, valid for hydrogenated $\mathrm{Ti}(\mathrm{Ti}-\mathrm{H})$ adatom on the oxygen site of $\mathrm{MgO}(001)$. In Sec. IV, we present our description of the $\mathrm{Ti}-\mathrm{H}$ adatom on $\mathrm{MgO}$ based on density functional theory (DFT) calculations and how this connects with the crystal field Hamiltonian presented in the previous section.

In Sec. V, we derive analytical expressions for the $g$ tensor anisotopy of Ti-H on $\mathrm{MgO}$, based on the model of Sec. III. The $g$ tensor obtained depends on the Ti spin orbit coupling and the crystal field parameters that can be obtained from DFT. In Sec. VI, we discuss how the $g$ factor can be modulated for $\mathrm{Ti}-\mathrm{H}$ on $\mathrm{MgO}$ by application of an electric field between tip and surface and we compute the associated Rabi energy. In Sec. VII, we briefly present the analogous piezoelectric modulation for $\mathrm{Fe}$ on $\mathrm{MgO}$. In Sec. VIII, we discuss how the contact hyperfine interaction become anisotropic due to the $g$-factor anisotropy and how the $g$-factor modulation could induce nuclear spin flip transitions. In Sec. IX, we discuss the role of both the $g$ tensor anisotropy of the adatom and the magnetic anisotropy of the tip in the efficiency of the exchange modulation [26] mechanism. In Sec. X, we show that the DC component of the tip-surface electric field induces a shift of the transition energy of the adatom due to the modification of the $g$ tensor. Finally, in Sec. XI, we present some limitations of our models and we list our main conclusions. The Appendixes describe technical steps of some results used in the main text.

\section{SPIN TRANSITIONS DRIVEN BY ANISOTROPIC MODULATION OF THE $g$ TENSOR}

For a free electron in vacuum, the interaction with a magnetic field is perfectly isotropic, in the sense that the energy splitting is the same regardless of the direction of the magnetic field $\vec{B}$. This results leads to the isotropic Zeeman interaction, $g \mu_{B} \vec{S} \cdot \vec{B}$. In contrast, for a general class of systems, the interplay between the spin orbit coupling $\vec{\ell} \cdot \vec{S}$, the orbital coupling to the magnetic field $\vec{\ell} \cdot \vec{B}$, and the crystal field splitting leads to an anisotropic Zeeman interaction. For instance, in the case of $S=1 / 2$ adatoms, such as Ti-H [6,8-11] and $\mathrm{Cu}$ [10] on a $001 \mathrm{MgO}$ surface, the interplay between the spin orbit coupling and the crystal field splitting leads to an anisotropic Zeeman interaction with different off-plane $(z)$ and in-plane $x y$ [29]:

$$
\mathcal{H}_{Z}=g_{x} \mu_{B} B_{x} S_{x}+g_{z} \mu_{B} B_{z} S_{z}=\mu_{B} \vec{b}_{0} \cdot \vec{S},
$$

where $\vec{b}_{0}=\left(g_{x} B_{x}, 0, g_{z} B_{z}\right)$.
As we show below, the tip $a c$ electric field modulates the $g_{x}$ and $g_{z}$ coefficients, resulting in a time-dependent perturbation:

$$
\mathcal{V}(t)=\left(\delta g_{x} \mu_{B} B_{x} S_{x}+\delta g_{z} \mu_{B} B_{z} S_{z}\right) \cos (2 \pi f t) .
$$

This equation can be written down as

$$
\mathcal{V}(t)=\cos (2 \pi f t) \mu_{B} \vec{b}_{1} \cdot \vec{S},
$$

where $\vec{b}_{1}=\left(\delta g_{x} B_{x}, 0, \delta g_{z} B_{z}\right)$. This perturbation can induce spin transitions between the two eigenstates of $\mathcal{H}_{0}$ if $\vec{b}_{0}$ and $\vec{b}_{1}$ are noncollinear, $\left|\vec{b}_{1} \times \vec{b}_{0}\right| \neq 0$. This yields

$$
\frac{\delta g_{z}}{g_{z}} \neq \frac{\delta g_{x}}{g_{x}} \text {. }
$$

Thus, the perturbation (2) induces spin transitions if the relative modulations of the $g$ factor are different. If we express the perturbation Hamiltonian in the basis of eigenstates of $\mathcal{H}_{Z}| \pm\rangle= \pm \frac{\Delta_{Z}}{2}| \pm\rangle$

$$
\mathcal{V}(t)=\Omega_{g} \cos (2 \pi f t)(|+\rangle\langle-|+|-\rangle\langle+|),
$$

where the Rabi coupling $\Omega_{g}$ is given by particularly simple equation, derived in Appendix A,

$$
\Omega_{g}=\frac{\Delta_{Z}}{4} \sin 2 \theta\left(\frac{\delta g_{z}}{g_{z}}-\frac{\delta g_{x}}{g_{x}}\right),
$$

where $\Delta_{z} \equiv \mu_{B}\left|\vec{b}_{0}\right|$ is the Zeeman splitting, then $\theta$ is the polar coordinate of $\vec{b}_{0}$ defined in Eq. (1) [see also Eqs. (A2), (A12), and (A13)]. From Eq. (6), we immediately infer that the Rabi coupling created by the modulation of the $g$ factor scales linearly with the magnitude of the magnetic field and has a very strong dependence on its orientation relative to the normal of the surface.

\section{A MODEL HAMILTONAN FOR Ti-H on MgO}

We now consider a model that describes a single electron occupying a $d$ shell with a crystal field splitting with $C_{4}$ symmetry for rotations in the $x y$ plane around the $z$ axis. This permits one to obtain closed analytical expressions for the $g$ tensor in terms of the crystal field parameters and the spin orbit coupling. In addition, our DFT calculations, discussed below, show that the model provides a fairly good description of hydrogenated $\mathrm{Ti}$ adatoms on the oxygen site of an $\mathrm{MgO}$ surface, relevant for STM-EPR experiments [6,8-11].

$\mathrm{Ti}^{2+}$ on $\mathrm{MgO}$ has two electrons in the $d$ shell, and our DFT calculations show it has $S=1$, in contrast with the experimental results $[6,8-11]$. It has been proposed that the reason why $\mathrm{Ti} / \mathrm{MgO}$ has $S=1 / 2$ is because it chemisorbs a hydrogen atom [6]. Our DFT calculations back up this assumption [6]. They show that hydrogen sits on top of Ti, almost colinear with the oxygen-Ti line that goes perpendicular to the surface. In that geometry, the $s$ orbital of $H$ hybridizes both with $d_{z^{2}}$ and $s$ orbitals of Ti and forms a molecular bonding-antibonding pair that hosts two electrons. This leaves only a single electron in the $d$ shell, that occupies the $x^{2}-y^{2}$ orbital, so that the Ti-H system effectively has $S=1 / 2$. We use the following Hamiltonian for the outermost electron of a single $S=1 / 2$ electron in a $d$ shell, that includes crystal field terms, spin orbit coupling, and Zeeman interaction:

$$
\begin{aligned}
\mathcal{H}_{0}= & -|D| \ell_{z}^{2}+F\left(\left(\ell^{(+)}\right)^{4}+\left(\ell^{(-)}\right)^{4}\right) \\
& +\lambda \vec{S} \cdot \vec{\ell}+\mu_{B} \vec{B} \cdot(g \vec{S}+\vec{\ell}) .
\end{aligned}
$$




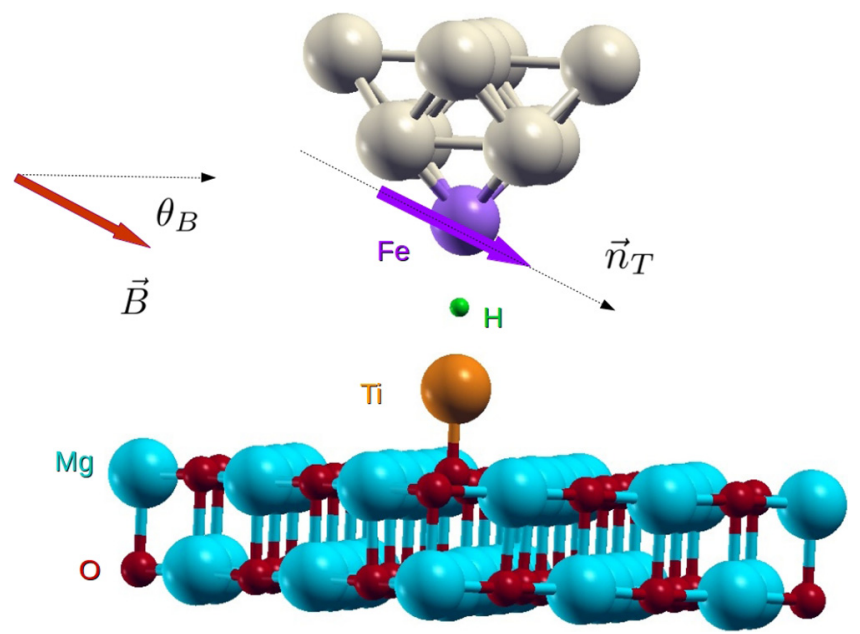

FIG. 1. Scheme of the STM-EPR experimental setup [3-12] with the atomic structure of Ti-H on the oxygen site of $\mathrm{MgO} . \vec{B}$ is the external magnetic field applied during the experiment (with an angle $\theta_{B}$ measured with respect to the surface) and $\vec{n}_{T}$ shows the direction of the magnetic moment of the tip. Red balls represent $\mathrm{O}$ atoms, blue balls are $\mathrm{Mg}$ atoms, the violet ball is for the Fe atom, the green ball indicates the $\mathrm{H}$ atom, and the orange one is for the Ti atom.

Here, $\ell$ is the single particle angular momentum operator for the $d$ electrons and $\vec{S}$ are the spin-1/2 matrices. Notice that Baumann et al. [3] used a mathematically similar expression for a multielectronic Hamiltonian multiplet with $L=S=2$, valid for $\mathrm{Fe}$ on $\mathrm{MgO}$. The crystal field terms account for the electrostatic interactions of the first neighbor charged ions of the Ti adatom (see Fig. 1). The $\mathrm{Mg}$ atoms are positively charged ions that reduce the energy of the $x y$ and $x^{2}-y^{2}$ orbitals compared to the $x z, y z$, and $\mathrm{z}^{2}$ orbitals. Oxygen atom is negatively charged and it increases the energy of the $z^{2}$ orbital. The $D$ term accounts for these effects. In addition, the $F$ term accounts for the $C_{4}$ symmetry of the surface and discriminates between the $x y$ and $x^{2}-y^{2}$ orbitals, as one of them points toward the positively charged $\mathrm{Mg}$ ions, reducing the energy of that orbital, whereas the other points toward the oxygen atoms. The lowest energy orbital should be the $x^{2}-y^{2}$ [if we take the oxygen atoms in the (10) and (01) directions].

\section{DENSITY FUNCTIONAL CALCULATIONS FOR HYDROGENATED Ti}

In this section, we focus on the electronic properties of individual hydrogenated $\mathrm{Ti}$ adatoms at $\mathrm{MgO}$ on top of oxygen, as described with density functional theory (DFT) calculations. With this aim, we have employed QUANTUM ESPRESSO [30], using projected augmented wave pseudopotentials, Perdew-Burke-Ernzerhof exchange correlation functional and 50-70 Ry of plane wave energy cutoff, as described elsewhere [30-32]. We performed calculations in a structure formed by a bilayer of $\mathrm{MgO}$, as shown in Fig. 1, consisting of $36 \mathrm{O}$ atoms (red balls) and $36 \mathrm{Mg}$ atoms (blue balls) together with the hydrogenated $\mathrm{Ti}$ (orange ball) with one $\mathrm{H}$ (green ball). In order to check some results, we also performed a few number of calculations using a bigger supercell with 64 $\mathrm{O}$ atoms, $64 \mathrm{Mg}$ atoms, and the hydrogenated Ti. The main

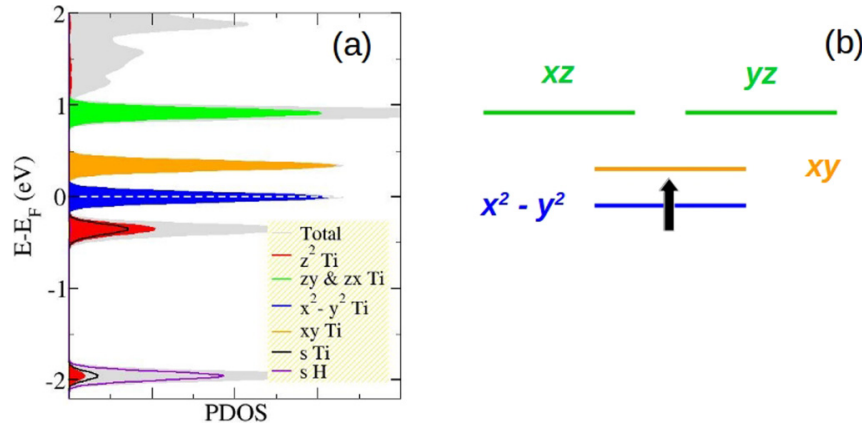

(b)

FIG. 2. (a) DFT calculations for the spin-unpolarized density of states projected over $d$ and $s$ orbitals of Ti and the $s$ orbital of hydrogen. The gray shadow shows the total density of states. (b) Energy level cartoon.

distortions created by the adatom in the $\mathrm{MgO}$ bilayer are (i) an upward displacement of the closest oxygen(s) to adatoms and (ii) a downward distortion of the $\mathrm{Mg}$ atoms located below the Ti-bonded oxygen atoms. Our DFT calculations found that a hydrogenated $\mathrm{Ti}$ atom shows $S=1 / 2[6,8]$. The spin density of hydrogenated $\mathrm{TiO}$ (with the $\mathrm{Ti}$ and $\mathrm{H}$ atoms located collinear along the $z$ axis) is consistent with a filling of the $d_{x^{2}-y^{2}}$ orbital, since we are assuming that the $\mathrm{Mg}$ atoms' first neighbor are in the $x$ and $y$ axes.

Figure 2(a) shows the projected density of states over $d$ orbitals for the hydrogenated $\mathrm{Ti}$ at $\mathrm{MgO}$ on top of oxygen, computed with no spin polarization. DFT yields $x^{2}-y^{2}$ and a hybrid orbital $z^{2}-s$ as the lowest energy orbitals, within the $d$ manifold. The $z^{2}$ of the Ti $d$ shell is strongly hybridized with the hydrogen $1 s$ orbital. As a result, the $z^{2}$ and the $s$ are split in energy and altogether host two electrons. The $x y$ orbital comes next in energy and is empty. The orbital doublet $x z$ and $y z$ lies higher up in energy. Calculations show that $x^{2}-y^{2}$ hosts exactly one electron. It is apparent that the Hamiltonian model Eq. (7) with $\ell_{z}= \pm 1, \pm 2$ describes the four orbitals $x^{2}-y^{2}, x y$, $x z$, and $y z$.

\section{A. Connection between DFT and model Hamiltonian}

We now explain how to obtain a rough estimate of $D$ and $F$ parameters that enter in the crystal field Hamiltonian (7). The method amounts to fit the energy difference of the peaks in the density of states obtained from a spin-unpolarized DFT calculation to those obtained from Eq. (7):

$$
\begin{gathered}
E_{x^{2}-y^{2}}-E_{x y}=48 F, \\
2 E_{x z}-E_{x^{2}-y^{2}}-E_{x y}=6|D|,
\end{gathered}
$$

Using these equations, from inspection of the density of states we infer the values $D \simeq-255 \mathrm{meV}$ and $F \simeq 7.50 \mathrm{meV}$. This crude approximation is enough for the scope of this work.

We can also obtain an estimate for the modulation of the crystal field parameters, $D$ and $F$, as the length of the Ti-O bond is changed from its equilibrium position. The calculation is carried out moving $\mathrm{Ti}$ atom and relaxing the four closest neighbor $\mathrm{Mg}$ atoms, the $\mathrm{O}$ atom below and the $\mathrm{H}$ atom, keeping all the others fixed. The results of the parameters $D$ 
and $F$, obtained with this procedure, allow us to obtain the following relation of $D, F$, and the strain:

$$
\begin{gathered}
\frac{d F}{d z}=-6 \frac{\mathrm{meV}}{\AA}, \\
\frac{d D}{d z}=+188 \frac{\mathrm{meV}}{\AA} .
\end{gathered}
$$

These values are used later on to estimate how the piezoelectric displacement of the Ti-O bond modulates the crystal field values $F$ and $D$ that in turn modulate the $g$ tensor.

\section{B. Calculation of the $g$ tensor from DFT}

We have calculated the $g$ tensor components from our DFT calculations using gauge including projector augmented waves (GIPAW). GIPAW is a DFT-based method to calculate magnetic resonance properties [33], where spin orbit coupling is implemented in a perturbative way. Our calculations for the structure in equilibrium $\delta z=0$ give us a diagonal $g$ tensor with components $g_{x}=g_{y}=1.974$ and $g_{z}=1.881$. As we discuss now, the model Hamiltonian Eq. (7) provides physical insight on the origin of the anisotropy and very good agreement with the values obtained from DFT.

\section{CALCULATION OF THE $g$ TENSOR FROM THE MODEL}

We now use the model Hamitlonian Eq. (7) to compute the $g$ tensor. We do this at two levels of approximation. First, we obtain analytical approximate expressions from the model Hamiltonian. Second, we obtain the $g$ tensor from the exact numerical solution of the model. Both the analytical and numerical approaches permit us to relate the $g$-tensor components with the crystal field parameters $D$ and $F$ and the spin orbit coupling $\lambda$. On account of the $C_{4}$ symmetry of the Hamiltonian, the $g$ tensor is diagonal and has $g_{x}=g_{y}$. Therefore, we only need to compute $g_{z}$ and $g_{x}$.

\section{A. Calculation of $g_{z}$}

We first consider the response of the electron in the $x^{2}-y^{2}$ state to a magnetic field in the $z$ direction. For that matter, we need to consider the space of four states with $\ell_{z}= \pm 2$ and $S_{z}= \pm 1 / 2$. Within this subspace, spin orbit coupling only acts through the $S_{z} \ell_{z}$ term. Therefore, $S_{z}$ is conserved, and the Hamiltonian for each $S_{z}$ is given by

$$
\mathcal{H}_{S_{z}}\left(B_{z}\right)=\left(\begin{array}{cc}
-\lambda \ell_{z} S_{z}+\Delta_{-}\left(B_{z}\right) & 24 F \\
24 F & \lambda \ell_{z} S_{z}+\Delta_{+}\left(B_{z}\right)
\end{array}\right),
$$

where $\Delta_{ \pm}\left(B_{z}\right)=\mu_{B} B_{z}\left(g S_{z} \pm \ell_{z}\right)$ with $\ell_{z}=2$. Hamiltonian Eq. (12) can be written as

$$
\mathcal{H}\left(S_{z}\right)=g \mu_{B} B_{z} S_{z}+\vec{h}\left(S_{z}\right) \cdot \vec{\sigma},
$$

where

$$
\vec{h}\left(S_{z}\right)=\left[24 F, 0,-\ell_{z}\left(\mu_{B} B_{z}+S_{z} \lambda\right)\right] .
$$

We thus have $\epsilon_{2}\left(S_{z}, \pm\right)=-4|D|+E^{( \pm)}\left(S_{z}\right)$, where

$$
E^{( \pm)}\left(S_{z}\right)=g \mu_{B} B_{z} S_{z} \pm \sqrt{(24 F)^{2}+\ell_{z}^{2}\left(\mu_{B} B_{z}+S_{z} \lambda\right)^{2}} .
$$

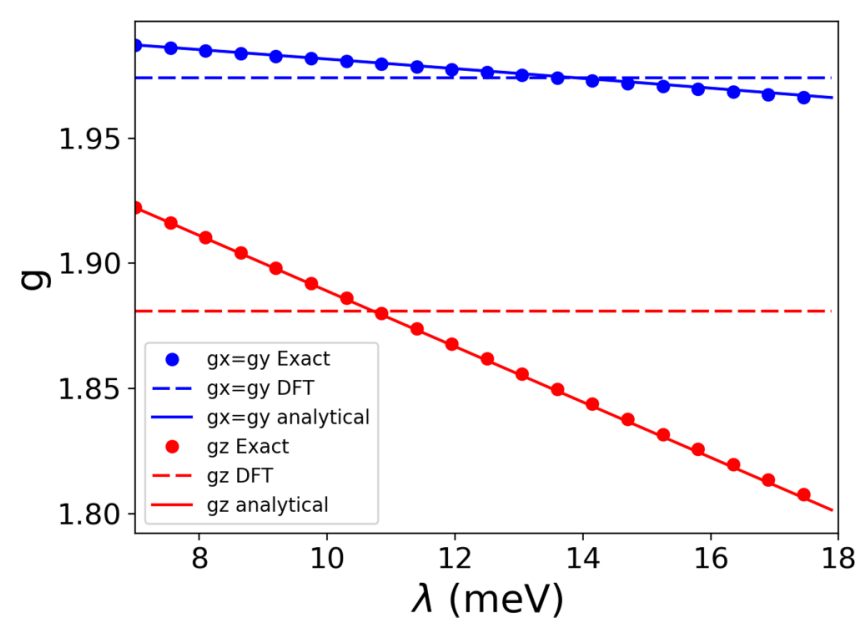

FIG. 3. Dependence of $g_{x}$ and $g_{z}$ on spin orbit coupling $\lambda$, for Ti-H on oxygen, obtained in two ways: solution of full model Eq. (7) with $D=-255 \mathrm{meV}$ and $F=14 \mathrm{meV}$ (symbols) and using analytical results ignoring $\ell_{z} \neq 2$ manifolds [Eqs. (15) and (22)] (lines). DFT results are shown for reference as dashed lines.

We now Taylor expand the ground state of the $E^{-}$states around $B_{z}=0$ :

$$
E^{(-)}\left(S_{z}\right)=-\sqrt{(24 F)^{2}+\lambda^{2}}+g \mu_{B} S_{z} B_{z}+\delta g_{z} S_{z} \mu_{B} B_{z},
$$

where

$$
g_{z}=g+\delta g_{z}=2-\frac{4 \lambda}{\sqrt{(24 F)^{2}+\lambda^{2}}} .
$$

Interestingly, there are no higher order corrections to $\delta g_{z}$, coming from mixing with the $\ell_{z}= \pm 1$ manifold. This is confirmed by the comparison of Eq. (15) with the results obtained from exact diagonalization of the complete model (7). As a result, we can use Eq. (15) for obtain the ratio $\frac{F}{\lambda}=$ 1.4 that gives $g_{z}$, in agreement with the DFT result $g_{z}=1.881$ The dependence of $g_{z}$ on $\lambda, F$, and $D$ is shown in Fig. 3. Note that, as shown in Fig. $4, g_{z}$ does not depend on $D$ and we can use $F / \lambda=1.4$ to ensure that $g_{z}=1.881$.

\section{B. Calculation of $g_{x}$}

We now obtain an analytical expression for $g_{x}$ for the $x^{2}-y^{2}$ ground-state manifold of Hamiltonian Eq. (12). For that matter, we represent the operator $\mu_{B} B_{x}\left(g S_{x}+\ell_{x}\right)$ in the basis of eigenstates of $\mathcal{H}(\sigma)$ :

$$
\begin{aligned}
& \left|\psi_{-}(\uparrow)\right\rangle=\cos \frac{\alpha}{2}|-2, \uparrow\rangle-\sin \frac{\alpha}{2}|+2, \uparrow\rangle, \\
& \left|\psi_{-}(\downarrow)\right\rangle=\cos \frac{\alpha}{2}|+2, \downarrow\rangle-\sin \frac{\alpha}{2}|-2, \downarrow\rangle,
\end{aligned}
$$

where the angle $\alpha$ is defined as

$$
\vec{h}\left(S_{z}\right)=\left|\vec{h}\left(S_{z}\right)\right|\left(\sin \alpha\left(S_{z}\right), 0, \cos \alpha\left(S_{z}\right)\right)
$$

and $\vec{h}$ is defined in Eq. (14). In this subspace, the matrix elements of $\ell_{x}$ are zero and the only nonzero matrix element of $S_{x}$ reads

$$
\left\langle\psi^{-}(\uparrow)\left|S_{x}\right| \psi^{(-)}(\downarrow)\right\rangle=-\frac{1}{2} \sin \alpha .
$$



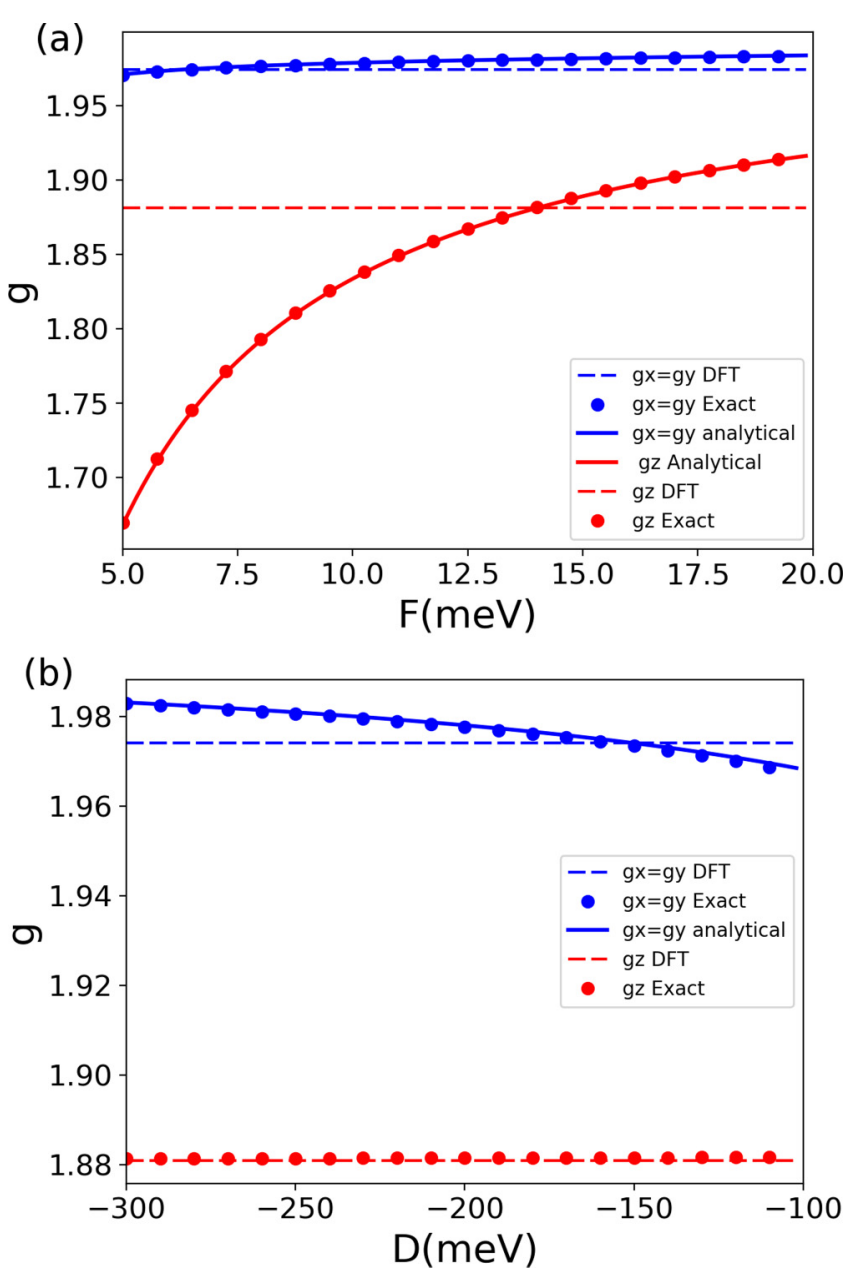

FIG. 4. Dependence of $g_{x}$ and $g_{z}$ on crystal field parameters $F$ (a) and $D$ (b) for Ti-H on oxygen, obtained from full model Eq. (7) (solid points in both panels), DFT (dashed lies), and analytically (solid lines) [Eqs. (15) and (22)]. In both panels, we take $\lambda=$ $10 \mathrm{meV}$. (a) $D=-255 \mathrm{meV}$. (b) $F=14 \mathrm{meV}$.

For $B_{z}=0$, we have

$\sin \alpha=\frac{24 F}{\sqrt{(24 F)^{2}+\lambda^{2}}}, \quad \cos \alpha=\frac{-\left|2 S_{z}\right| \lambda}{\sqrt{(24 F)^{2}+\lambda^{2}}}$.

Thus, the eigenvalues of the Hamiltonian are

$$
\epsilon_{2}\left(B_{x}\right)=-3|D|-\sqrt{(24 F)^{2}+\lambda^{2}} \pm \frac{g}{2} \mu_{B} B_{x} \sin \alpha .
$$

We thus have

$$
g_{x}^{(1)}=g \sin \alpha=g \frac{24 F}{\sqrt{(24 F)^{2}+\lambda^{2}}} .
$$

We now consider the contributions to $g_{x}$ that arise from the virtual transitions to the $\ell= \pm 1$ levels. These are driven by the combined action of the $\mu_{B} \ell_{x} B_{x}$ and the flip-flop part of the spin orbit interaction. This additional contribution gives

$$
\delta g_{x}^{(2)}=-\frac{2 \lambda}{3|D|+24|F|+|\lambda|}
$$

so that the $g_{x}$ factor is given by

$$
g_{x}=g \frac{24 F}{\sqrt{(24 F)^{2}+\lambda^{2}}}-\frac{2 \lambda}{3|D|+24|F|+|\lambda|} .
$$

The anisotropy of the $g$ tensor arises ultimately from the fact that the $\ell_{z}= \pm 2$ states have a strong additional orbital response only when $B$ is applied in the $z$ direction. This extra contribution is quenched by the $F$ crystal field term that leads to states with equal weight on the two $\ell_{z}= \pm 2$ states but promoted by the spin orbit coupling. The resulting anisotropy is thus controlled by the competition between $\lambda$ and $F$. In addition, $g_{x}$ has also a contribution that arises from virtual coupling to the $\ell_{z}= \pm 1$ states. For the values of $D, F, \lambda$ adequate to describe $\mathrm{Ti}-\mathrm{H}$ on $\mathrm{MgO}$, the dominant contribution to the deviation of $g_{x}$ from the value $g=2$ arises from the virtual coupling to $\ell= \pm 1$.

So, for $F=0$ we have $g_{x}^{(1)}=0$, because the spin orbit coupling correlates $S_{z}$ and $\ell_{z}$ so that spin flips entail momentum flips that are forbidden, and $g_{z}=-2$ because of the dominant orbital contribution. In the opposite limit of $\lambda=0$, we recover $g_{x}=g_{z}=2$. If we repeat the analysis for $g_{y}$, we obtain $g_{x}=g_{y}$, as expected from the $\mathrm{C}_{4}$ surface's symmetry.

In Figs. 3 and 4, we show the predictions for $g_{x}$ and $g_{z}$, as functions of $\lambda, D$, and $F$, obtained using both the analytical formulas Eqs. (15) and (22) and the exact solution of the complete Hamiltonian Eq. (7). The DFT results are shown as dashed horizontal lines. In Figs. 3 and 4(a) we take $D=$ $-255 \mathrm{meV}$, roughly estimated from DFT, using Eq. (9), which gives a single particle spectrum, in agreement with the results of DFT. In Fig. 4(b), we take $\lambda=10 \mathrm{meV}$ and $F=14 \mathrm{meV}$, also inferred from comparison with DFT. In Fig. 4 , we fix $\lambda=10 \mathrm{meV}$ and $F=1.4 \lambda$ so that we obtain values very close to those obtained with DFT. Finally, Fig. 4(b) shows that the dependence of $g_{x}$ on $D$ is small and $g_{z}$ does not depend on $D$.

Summing up, the results of this section show how, for a model with the symmetry adequate for a $\mathrm{TiH}$ on top of an oxygen on an (001) $\mathrm{MgO}$ surface, the $g$ factor is anisotropic, $g_{z} \neq g_{x}=g_{y}$, and how $g_{z}$ and $g_{x}$ depend on the crystal field parameters $F$, and to a lesser extent, on $D$. Our analytical model is able to give $g_{x}$ and $g_{z}$, in agreement with the values obtained from DFT.

\section{PIEZOELECTRIC MODULATION OF $g$ FOR Ti-H on MgO}

We have shown that a modulation of the $g$-factor anisotropy would induce spin-flip transitions [Eq. (6)], and we have computed how the $g$-factor components depend on the crystal field parameters $F$ and $D$. We now argue that an electric field applied perpendicular to the surface of $\mathrm{MgO}$ modulates $F$ and $D$, and thereby the $g$-factor anisotropy, resulting in spin transitions between the two states of the lowest energy Kramers doublet of Eq. (7).

Our DFT calculations show that crystal field parameters $D$ and $F$ are functions of the adatom-oxygen distance, $z$ : $D(z), F(z)$ [see Eqs. (10) and (11) and Fig. 5]. We denote the equilibrium position by $z_{\text {eq }}$. The electric field across the gap between the STM and the MgO surface, $E=V_{\text {tip }} / d$, where $d$ is the tip-MgO distance, induces a force on the adatom, $F=$ $q_{\text {adatom }} V_{\text {tip }} / d$ on account of its charge $q_{\text {adatom }}[3,11,26]$. This 


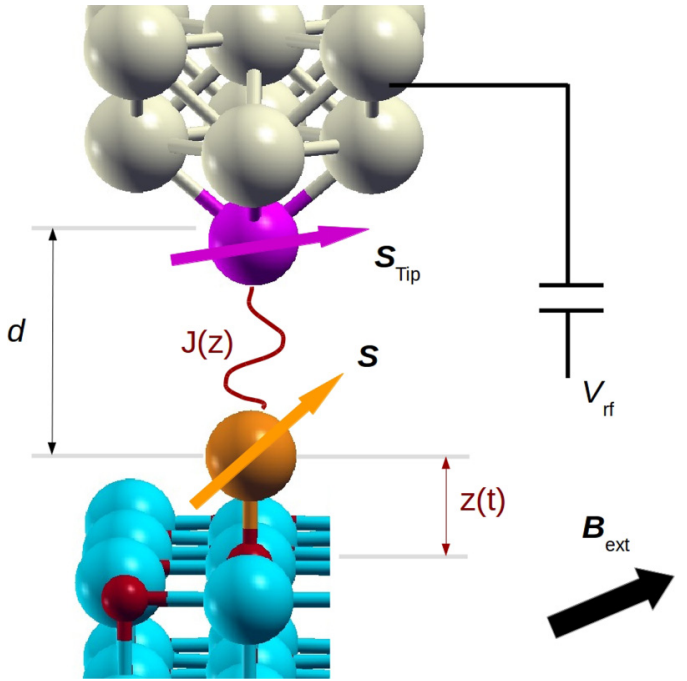

FIG. 5. Schematic showing a magnetic atom (orange) on $\mathrm{MgO}$ and an Fe atom (violet) attached to the apex of the STM tip. The solid (orange and violet) arrows indicate the orientations of the respective magnetic moments $\left(\vec{S}\right.$ and $\left.\overrightarrow{S_{\text {tip }}}\right)$. The exchange interaction $J$ is indicated by the red curve and the piezoelectric displacement is represented with a red arrow. A radio-frequency voltage drives the ESR of the magnetic atom. The external magnetic field $B_{\text {ext }}$ is applied out of the plane of the substrate (with a small angle).

force is compensated by a restoring elastic force $F=-k \delta z$. Thus, the adatom equilibrium position is displaced by [26]

$$
\delta z(t)=\frac{q_{\text {adatom }} V_{\text {tip }}(t)}{k d} .
$$

This equation is valid for a time-dependent $V_{\text {tip }}$ as long as its Fourier components are away from the mechanical resonance frequency of the stretching mode, $\sqrt{\frac{k}{M}}$, where $M$ is the mass of the adatom. According to our DFT calculations [11,26], this frequency is up in the $\mathrm{THz}$ range, as long as we ignore the contributions coming from the off-plane (flexural) phonons of the $\mathrm{MgO}$. In the following, we assume $V_{\text {tip }}(t)=V_{\mathrm{RF}}^{0} \cos (2 \pi f t)$ so that we have

$$
\delta z(t)=\frac{q_{\text {adatom }} V_{\mathrm{RF}}^{0}(t)}{k d} \cos (2 \pi f t) \equiv \delta z_{0} \cos (2 \pi f t) .
$$

From our DFT calculations for $\mathrm{Ti}-\mathrm{H}$ on $\mathrm{MgO}$ [11], we obtain $k=290 \mathrm{eV} \mathrm{nm}^{-2}$, so that for RF tip voltages values ranging from $e V_{\mathrm{RF}}^{0}=10 \mathrm{meV}$ to $e V_{\mathrm{RF}}^{0}=20 \mathrm{meV}$ and $d=5 \AA$, the piezoelectric displacement amplitude goes from $\delta z_{0}=$ $0.07 \mathrm{pm}$ to $\delta z_{0}=0.14 \mathrm{pm}$.

The modulation of crystal field parameters $F$ and $D$ with the Ti-O bond length leads to a modulation of the $g$ tensor:

$$
\delta g_{a}=\left(\frac{\partial g_{a}}{\partial F} \frac{\partial F}{\partial z}+\frac{\partial g_{a}}{\partial D} \frac{\partial D}{\partial z}\right) \delta z(t) .
$$

It must be noted that this equation is also valid in the DC limit.

We now proceed to estimate the magnitude of the Rabi coupling associated to the modulation of the $g$ factor. We do that using two different methods that, as we discuss below, give the same result. The first method consists of using Eq. (6). In the second method, we evaluate directly the matrix elements
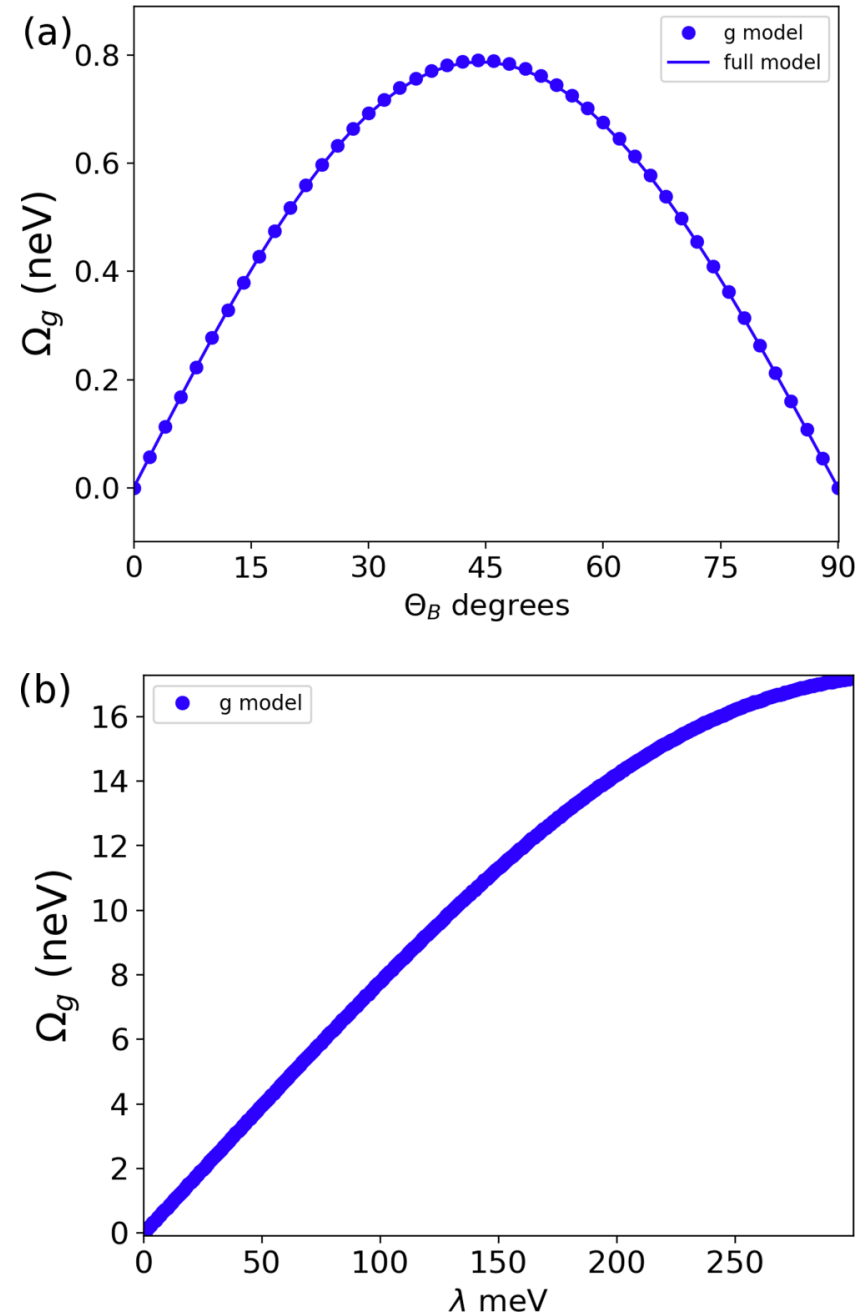

FIG. 6. (a) Rabi coupling due to $g$-factor modulation as a function of the angle $\theta_{B}$ between $\vec{B}$ and surface calculated with two methods: Eqs. (6) and (27). $\lambda=10 \mathrm{meV}, F=1.4 \lambda=14 \mathrm{meV}, D=$ $-255 \mathrm{meV}, B=1 T, V_{F}=20 \mathrm{meV}$, STM-surface distance $d=5 \AA$, $k=290 \mathrm{eV} / \mathrm{nm}^{2}$. (b) Dependence of $\Omega_{g}$ on $\lambda$, keeping all the other constants the same and $\theta_{B}=45 \mathrm{deg}$.

of the crystal field operators, using the same approach used in previous works $[3,26]$.

\section{A. Rabi coupling from the $g$-factor anisotropy}

In order to compute the Rabi coupling from Eq. (6), we need to compute Eq. (25). For that matter, we obtain $\frac{\partial g_{a}}{\partial F}$ and $\frac{\partial g_{a}}{\partial D}$ from our model Hamiltonian, and we use $\frac{\partial F}{\partial z}, \frac{\partial D}{\partial z}$ calculated from DFT calculations in Sec. IV A.

We are now in position to estimate $\Omega_{g}$, combing Eqs. (6), (10), (24), and (25). We now take $V_{\mathrm{RF}}^{0}=20 \mathrm{meV}, d=5 \AA$, and $k=290 \mathrm{eV} / \mathrm{nm}^{2}$ (taken from DFT calculations). This yields a strain of the Ti-O distance of $\delta z_{0}=0.14 \mathrm{pm}$. In Fig. 6(a), we plot the magnitude of the Rabi coupling so obtained, as a function of the angle between the magnetic field and the surface, $\theta_{B}$ for $B=1$ Tesla. The first thing to note is that the magnitude of $\Omega_{g}$ is between one and two orders of magnitude smaller than the experimental values reported in our previous work [11]. Therefore, other mechanism, most 
likely exchange modulation [26], has to be involved in the electric field driving of the spin for EPR-STM for Ti-H/MgO.

The magnitude of $\Omega_{g}$ scales linearly both with the applied field $B$ and with the RF electric field $\frac{V_{\mathrm{RF}}}{d}$. The optimal angle to maximize $\Omega_{g}$ is close to $45 \mathrm{deg}$. In contrast, the exchange mechanism is independent of $B$ and scales exponentially [11] with $d$. Whereas the $g$-factor modulation is not dominant for $\mathrm{Ti}-\mathrm{H}$ on $\mathrm{MgO}$, it could be the dominant factor in heavier adatoms. To show this, in the bottom panel of Fig. 6(b) we plot $\Omega_{g}$ ramping $\lambda$, keeping all the other parameters the same and taking $\theta_{B}=45$. It is apparent that, for a wide range, $\Omega_{g}$ scales linearly with spin orbit coupling. Expectedly, $\Omega_{g}$ vanishes for $\lambda=0$, as the $g$-factor anisotropy is driven by $\lambda$.

\section{B. Rabi coupling from crystal field matrix elements}

We now carry out a sanity check. Given that the electronic spin flip transitions described by the effective $S=1 / 2$ model of Eqs. (1) and (2), arise ultimately from the modulation of the crystal field operators of the parent Hamiltonian of Eq. (7), we have computed the Rabi coupling using the parent model as well. To do so, we first obtain the two lowest eigenstates of Hamiltonian from Eq. (7), $\mathcal{H}| \pm\rangle=\left(E_{g} \pm \frac{\Delta_{z}}{2}\right)| \pm\rangle$ and we then compute the matrix elements of the perturbation operator:

$$
\mathcal{V}(t)=\frac{\partial D}{\partial z} \delta z(t) \ell_{z}^{2}+\frac{\partial F}{\partial z} \delta z(t)\left[\left(\ell^{(+)}\right)^{4}+\left(\ell^{(-)}\right)^{4}\right],
$$

where the time dependence is described by Eq. (24). We thus define

$$
\begin{aligned}
\Omega_{\mathrm{CF}}= & \frac{\partial D}{\partial z} \delta z_{0}\left\langle+\left|\ell_{z}^{2}\right|-\right\rangle \\
& +\frac{\partial F}{\partial z} \delta z_{0}\left\langle+\left|\left[\left(\ell^{(+)}\right)^{4}+\left(\ell^{(-)}\right)^{4}\right]\right|-\right\rangle .
\end{aligned}
$$

The results of the calculation of $\Omega_{\mathrm{CF}}$ as function of $\theta_{B}$, obtained with the same values of $\lambda, D, F, d, V_{\mathrm{RF}}, k$, and $B$ of the previous subsection, are shown in Fig. 6 and, expectedly, are in full agreement with those obtained using Eq. (6). This agreement validates our analysis.

\section{VII. $g$-FACTOR MODULATION OF Fe on MgO}

The results of the last paragraph show that it is possible to interpret the modulation spin driving coming from the modulation of the crystal field parameters [Eq. (27)] in terms of a modulation of the $g$ factor [Eq. (6)]. In the seminal work of Baumann et al. [3], the modulation of the crystal field (CF) was proposed as the driving mechanism for EPR-STM of $\mathrm{Fe} / \mathrm{MgO}$. Here we address the question of whether we can recast the $\mathrm{CF}$ mechanism in terms of the $g$-factor modulation, for the case of $\mathrm{Fe}$ on $\mathrm{MgO}$ as well.

In order to find the answer, that turns out to be negative, we need to model the $g$-factor modulation for $\mathrm{Fe}$ on $\mathrm{MgO}$ and to compare with the results obtained from the $\mathrm{CF}$ modulation. The main difference with the case of $\mathrm{Ti}-\mathrm{H}$ is that the ground state of $\mathrm{Fe}$ on $\mathrm{MgO}$ has $S=2$. Therefore, a multielectronic description is necessary [3,26,34].

We follow our own work [26] and we model Fe on $\mathrm{MgO}$ with a two levels of complexity. First, a microscopic Hamiltonian for six electrons in the $d$ orbitals of Fe, in the presence of a crystal field, spin orbit coupling, Coulomb interaction, and Zeeman interaction:

$$
\mathcal{H}_{\mathrm{Fe}}=H_{\mathrm{CF}}+H_{\mathrm{SOC}}+H_{\mathrm{Z}}+V_{e e} .
$$

The single particle crystal field Hamiltonian reads

$$
\mathcal{H}_{\mathrm{CF}}=D \ell_{z}^{2}+F\left(\ell_{x}^{4}+\ell_{y}^{4}\right) \text {. }
$$

As explained in Appendix C, this $\mathrm{CF}$ Hamiltonian turns is almost identical to the one we have used in Eq. (7) $\mathrm{Ti}-\mathrm{H} / \mathrm{MgO}$. As we did in the case of $\mathrm{Ti}-\mathrm{H} / \mathrm{MgO}$, we can infer $D$ and $F$ from DFT calculations [26]. For $z=z_{\text {eq }}$ we obtain, from DFT calculations, $F=-10 \mathrm{meV}$ and $D=-290 \mathrm{meV}$ [26]. The spin orbit coupling constant for $\mathrm{Fe}$ is $\lambda=35 \mathrm{meV}$ [26].

The many-body Hamiltonian can be solved exactly, by numerical diagonalization in a space made with all the states that accommodate six electrons in five spin-degenerate $d$ orbitals. The lowest energy manifold has five states, corresponding to a ground state with $S=2$ and can be described in terms of an effective spin model:

$$
H_{\mathrm{eff}}=-\mathcal{D}_{2} S_{z}^{2}+\mathcal{D}_{4} S_{z}^{4}-\mathcal{F}\left(S_{+}^{4}+S_{-}^{4}\right)+\mu_{B} \mathbf{B} \cdot g \cdot \mathbf{S},
$$

where the spin operators act on the $S=2$ subspace. The main difference with the $S=1 / 2$ case is the presence of single-ion anisotropy terms. The anisotropy terms $\mathcal{D}_{2}, \mathcal{D}_{4}, \mathcal{F}$, and the $g$ tensor can be obtained from the diagonalization of Hamiltonian Eq. (28). We obtain $\mathcal{D}_{2}=4.9 \mathrm{meV}, \mathcal{D}_{4}=0.23 \mathrm{meV}$, and $\mathcal{F}=11 \mathrm{neV}$. With these numbers, the spectrum of the $S=2$ manifold has a EPR active space formed by a doublet of states with $S_{z}= \pm 2$, that we denote as $|0\rangle$ and $|1\rangle$. Yet, this doublet is fundamentally different [24] from the $S=1 / 2$ Kramers pair, as it has a zero field splitting, given by $\Delta=48 \mathcal{F}=$ $0.5 \mu \mathrm{eV}$, due to quantum spin tunneling [35-38]. Thus, the EPR active doublet for $\mathrm{Fe}$ on $\mathrm{MgO}$ cannot be described in terms of a Zeeman-only Hamiltonian. At $B=0$, none of the two lowest energy states has a magnetic moment [38]. However, application of a modest off-plane field is enough to induce an off-plane magnetic moment in the two lowest energy states, on account of the small value of $\Delta$.

Diagonalizations of the multielectronic Hamiltonian Eq. (28) at finite magnetic field permit us to derive the $g$ tensor. Expectedly for a system with $C_{4}$ symmetry, it is diagonal in the Cartesian basis. The values of the $g$ tensor do depend on the single particle crystal field parameters $D$ and most notably on $F$ and $\lambda$. For the values quoted above, we obtain $g_{z}=2.8$ and $g_{x}=g_{y}=2.0$.

Importantly, all the constants in the effective Hamiltonian Eq. (30) do depend strongly on the single particle crystal field parameter $F$ that in turns depends on the piezoelectric displacement [26]. Following an argument similar to the one used for $d^{1}$ atoms, we can calculate the Rabi frequency derived from the effective Hamiltonian. We break it down in two types of terms:

$$
\Omega_{\mathrm{eff}}=\Omega_{\mathrm{ZFS}}+\Omega_{g} .
$$

The first comes from the modulation of the zero field energy constants, $\mathcal{D}_{2}, \mathcal{D}_{4}$, and $\mathcal{F}$ and was absent in the case of $S=$ $1 / 2$ adatoms. The dominant contributions [26] arise from the modulation of the $F$ term in the single particle crystal field of 


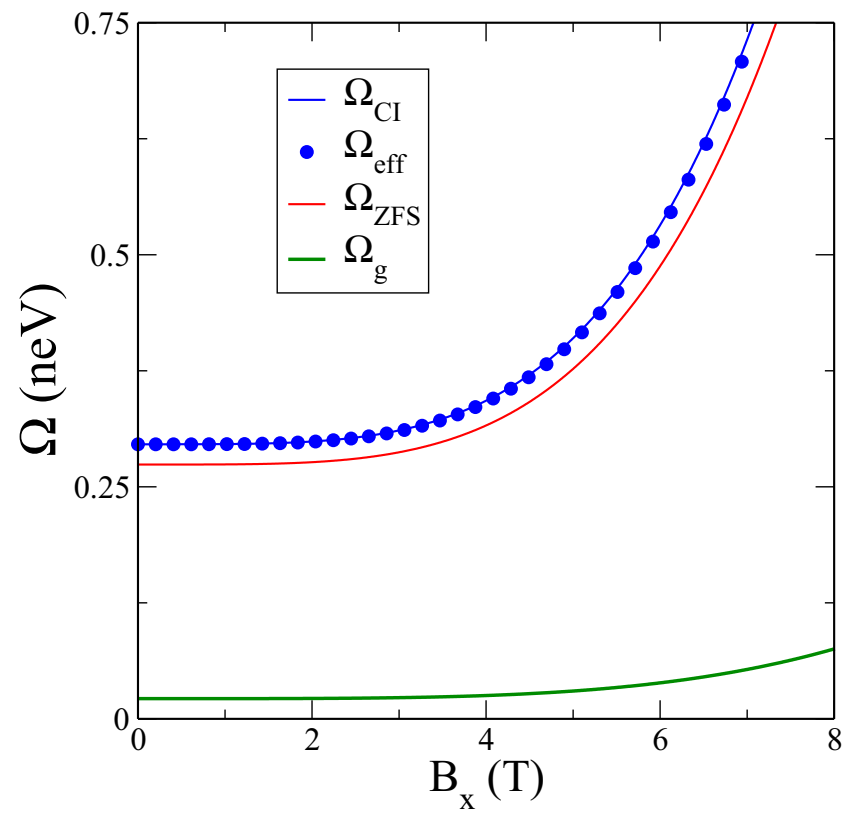

FIG. 7. Breakdown of Rabi coupling for $\mathrm{Fe}$ on $\mathrm{MgO}$ as a function of in-plane magnetic field $B_{x}$. The calculation were performed for $B_{z}=0.2 \mathrm{~T}, k=600 \mathrm{eV} / \mathrm{nm}^{2}, d=0.6 \mathrm{~nm}, V_{\mathrm{RF}}=8 \mathrm{mV}, D=$ $-290 \mathrm{meV}, F=-10 \mathrm{meV}, \lambda=35 \mathrm{meV}$, and $q=2 e$.

Eq. (29):

$$
\begin{aligned}
\Omega_{\mathrm{ZFS}}= & \left(-\frac{\partial \mathcal{D}_{2}}{\partial F}\left\langle 0\left|S_{z}^{2}\right| 1\right\rangle+\frac{\partial \mathcal{D}_{4}}{\partial F}\left\langle 0\left|S_{z}^{4}\right| 1\right\rangle\right. \\
& \left.-\frac{\partial \mathcal{F}}{\partial F}\left\langle 0\left|S_{+}^{4}+S_{-}^{4}\right| 1\right\rangle\right) \frac{\partial F}{\partial z},
\end{aligned}
$$

where $\frac{\partial F}{\partial z}=280 \mathrm{meV} / \mathrm{nm}$, obtained from DFT in a previous publication [26]. The second class of contribution to the Rabi coupling comes from the $g$-factor modulation, very much like the $S=1 / 2$ case:

$$
\Omega_{g}=\mu_{B}\left(\frac{\partial g_{z}}{\partial F} B_{z}\left\langle 0\left|S_{z}\right| 1\right\rangle+\frac{\partial g_{x}}{\partial F} B_{x}\left\langle 0\left|S_{x}\right| 1\right\rangle\right) \frac{\partial F}{\partial z} .
$$

We can assess the relative contribution of the zero field splitting and the g-tensor modulations in the following way. We first compute the Rabi coupling using the whole multielectron Hamiltonian [26] and we refer to this as $\Omega_{\mathrm{CI}}$. The calculation, done for $B_{z}=0.2 \mathrm{~T}, k=600 \mathrm{eV} / \mathrm{nm}^{2}, d=0.6 \mathrm{~nm}$, $V_{\mathrm{RF}}=8 \mathrm{mV}, D=-290 \mathrm{meV}, F=-10 \mathrm{meV}, \lambda=35 \mathrm{meV}$, and $q=2 e$ is shown in Fig. 7 as a function of the in-plane field $B_{x}$, together with the different contributions, $\Omega_{\mathrm{ZFS}}$ and $\Omega_{g}$, computed using the effective Hamiltonian Eqs. (30), (32), and (33). It is apparent that the $\Omega_{\mathrm{CI}}=\Omega_{\mathrm{ZFS}}+\Omega_{g}$, which validates our methods.

Importantly, our calculations show that the modulation of the $g$ factor is not a dominant contribution to the spin transitions driven by the modulation of the crystal field parameter $F$ due to off-plane piezoelectric distortion of the adatom. In addition, it was found in Ref. [26] that the exchange modulation mechanism is probably dominant for Fe. Therefore, the $g$-factor modulation plays a marginal role in the case of $\mathrm{Fe}$.

\section{MODULATION OF THE HYPERFINE INTERACTION}

Here we briefly address how the modulation of the $g$ factor anisotropy affects the hyperfine interaction. Recently, electrical control of an individual nuclear spin of $S=1 / 2 \mathrm{Cu}$ atom was demonstrated using STM-EPR [10]. For simplicity, here we consider the case of $\mathrm{Ti}-\mathrm{H}$ on $\mathrm{MgO}$, for which hyperfine splittings have been observed experimentally [8]. We consider a simplified hyperfine model where only the contact interaction term is considered:

$$
\mathcal{V}_{\mathrm{HF}}=A \mathbf{I} \cdot \mathbf{S} .
$$

For simplicity, the dipolar and quadruple terms are neglected, although they are known to be relevant for $\mathrm{Ti}-\mathrm{H}$ on $\mathrm{MgO}$ [8]. We now address how the modulation of the $g$-factor anisotropy affects this Hamiltonian and we find that the effective hyperfine coupling becomes anisotropic.

For that matter, we consider the representation of the isotropic hyperfine operator Eq. (34) in the basis set defined by the tensor product of the lowest energy eigenstates of Hamiltonian Eq. (7), whose wave functions are given by Eq. (16), and the eigenstates of the nuclear spin operator $I_{z}$. The resulting Hamiltonian reads

$$
\mathcal{H}_{\mathrm{HF}}=A_{\|} I_{z} S_{z}+A_{\perp}\left(I_{x} S_{x}+I_{y} S_{y}\right),
$$

where

$$
A_{\|}=A, \quad A_{\perp}=A \sin \alpha=A \frac{24 F}{\sqrt{(24 F)^{2}+\lambda^{2}}},
$$

where $F$ and $\lambda$ are the crystal field and spin orbit coupling in Eq. (7). It is apparent that the modification of the hyperfine interaction is connected to the $g$-factor anisotropy.

We now discuss how the modulation of the $g$ factor could lead to nuclear spin transitions that preserve the electronic spin. We consider the situation where a magnetic field induces an electronic Zeeman splitting so that the eigenstates of the electronuclear Hamiltonian can be split in two groups, according to their electronic spin [10]. We are interested in transitions between the low-energy manifold, so that initial and final states belong to the low-energy group, and only the nuclear spin changes in the transition. We thus consider transitions between two eigenstates that differ by single nuclear spin flip [10]:

$$
\left|I_{z}\right\rangle_{-}=\sqrt{1-\epsilon^{2}}|\downarrow\rangle\left|I_{z}\right\rangle+\epsilon|\uparrow\rangle\left|I_{z}-1\right\rangle
$$

and

$$
\left|I_{z}-1\right\rangle_{-}=\sqrt{1-\epsilon^{2}}|\downarrow\rangle\left|I_{z}-1\right\rangle+\epsilon|\uparrow\rangle\left|I_{z}-2\right\rangle,
$$

where $\epsilon \propto \frac{A_{\perp}}{g_{z} \mu_{B} B_{z}} \ll 1$. These states have a dominant $\downarrow$ electronic spin component and a small mixing due to the nonresonant spin-flip hyperfine interaction.

It is apparent that a perturbation that flips the electronic spin can induce transitions between these two states:

$$
{ }_{-}\left\langle I_{z}-1\left|S^{-}\right| I_{z}\right\rangle_{-} \propto \epsilon \sqrt{1-\epsilon^{2}} \simeq \frac{A_{\perp}}{g_{z} \mu_{B} B_{z}},
$$

showing the electronically driven nuclear spin transition matrix element is proportional to the hyperfine interaction. Thus, the same modulation of the $g$ tensor that drives electronic spin 
transitions, when $f$ in the range of the electronic Zeeman transition, can also drive nuclear-spin flip transitions if $f$ is in the range of the hyperfine interaction, as shown experimentally in the case of $\mathrm{Cu}$ on $\mathrm{MgO}$ [10].

We finally note that the modulation of $F$ will in turn change $A_{\perp}$ providing a time-dependent electron-nuclear perturbation,

$$
\delta \mathcal{V}_{\mathrm{HF}}=\delta A_{\perp}(t)\left(I_{x} S_{x}+I_{y} S_{y}\right),
$$

where $\delta A_{\perp}=\frac{\partial A_{\perp}}{\partial F} \frac{\partial F}{\partial z} \delta z(t)$. However, this electron-nuclear flip-flop operator cannot mix the states (37) and (38). The flip-flip modulation can induce EPR-like transitions, between state (37) and

$$
\left|I_{z}-1\right\rangle_{+}=\sqrt{1-\epsilon^{2}}|\uparrow\rangle\left|I_{z}-1\right\rangle+\epsilon|\downarrow\rangle\left|I_{z}\right\rangle .
$$

This could be a relevant mechanism for EPR-STM in systems with very large hyperfine interaction, such as $\mathrm{Bi}$ in silicon [39] or perhaps $\mathrm{Cu} / \mathrm{MgO}$ [11]. Hyperfine-driven electric spin dipole resonances have been reported in semiconductor quantum dots [40].

\section{ROLE OF ANISOTROPIC $g$ FACTOR AND THE EXCHANGE-DRIVEN MECHANISM FOR EPR-STM}

Although the main scope of this paper is to propose another mechanism for the electric field driving of the surface spins in STM-EPR, we briefly comment here on the role that the $g$-factor anisotropy plays on the exchange-modulation mechanism that we proposed in Ref. [26] and has been experimentally observed [11] for $\mathrm{Ti}-\mathrm{H}$ adatoms on $\mathrm{MgO}$. We now consider a Hamiltonian for the surface spin that, in addition to the Zeeman term, given by Eq. (1), has also exchange interaction with the tip. The magnetic moment of the tip is described semiclassically $[11,26]$, so that the Hamiltonian for the surface spin reads

$$
\mathcal{H}_{\mathrm{ex}}=\mathcal{H}_{Z}+J(z) \vec{n}_{T} \cdot \vec{S}
$$

where (see Figs. 1 and 5)

$$
\vec{n}_{T}=\left(\cos \left(\theta_{B}+\delta\right), 0, \sin \left(\theta_{B}+\delta\right)\right)
$$

describes the orientation of the tip moment, $\vec{B}=$ $B_{0}\left(\cos \left(\theta_{B}\right), 0, \sin \left(\theta_{B}\right)\right)$ is the external magnetic field forming and angle $\theta_{B}$ with the $\mathrm{MgO}$ surface as shown in Fig. 1 and $J(z)$ is the tip-adatom exchange interaction (Fig. 5) that depends on the tip-surface distance $z$.

In Appendix A 2, we derive an expression for the Rabi energy associated to the modulation $\delta J$ of the exchange formula:

$$
\begin{aligned}
\Omega_{J}= & \mathcal{E}_{J}\left(\frac{\Delta g_{x}}{g}-\frac{\Delta g_{z}}{g}\right) \cos \theta_{B} \sin \theta_{B} \cos \delta \\
& +\mathcal{E}_{J}\left(1+\frac{\Delta g_{x}}{g} \cos ^{2} \theta_{B}+\frac{\Delta g_{z}}{g} \sin ^{2} \theta_{B}\right) \sin \delta,
\end{aligned}
$$

where we write the ansotropic $g$ factor as

$$
\begin{gathered}
g_{x}=g+\Delta g_{x}, \\
g_{z}=g+\Delta g_{z},
\end{gathered}
$$

where $\Delta g_{x}$ and $\Delta g_{z}$ are the static contributions to the $g$-factor anisotropy,

$$
\mathcal{E}_{J} \equiv \frac{\delta J}{2 \Delta_{z}^{*}} g \mu_{B} B,
$$

and

$$
\Delta_{Z}^{*} \equiv \sqrt{\left(g_{x} \mu_{B} B_{x}+J n_{x}\right)^{2}+\left(g_{z} \mu_{B} B_{z}+J n_{z}\right)^{2}} .
$$

Let us consider now two different limits for this complicated formula. We study first the case $\delta=0$, i.e., when the tip magnetic moment is aligned with the external magnetic field. This amounts to assume that the tip magnetic moment has an isotropic $g$ factor. For $\delta=0$, the exchange modulation Rabi splitting reads

$$
\Omega_{J}=\frac{\mathcal{E}_{J}}{2} \sin 2 \theta_{B}\left(\frac{\Delta g_{x}}{g}-\frac{\Delta g_{z}}{g}\right) .
$$

Thus, this equation makes it apparent that the $g$-factor anisotropy of the surface spin is essential if the tip spin is aligned with $B(\delta=0)$. We note that, in spite of the similar aspect of Eqs. (6) and (48), they describe different mechanisms. In Eq. (6), the transitions are driven by the anisotropic modulation $\delta g$ of the $g$-factor anisotropy. In Eq. (48), the transitions are driven by the modulation of the tip-surface spin $\delta J$, but are enabled by the static $g$-factor anisotropy, given by $\Delta g$.

We now consider the case $\Delta g_{x}=\Delta g_{z}=\Delta g$, i.e., when the surface spin has an isotropic $g$ factor. We obtain

$$
\Omega_{J}=\mathcal{E}_{J}\left(1+\frac{\Delta g}{g}\right) \sin \delta .
$$

Thus, the exchange-modulation Rabi depends on the misalignment angle between the tip moment and the applied field. In both cases, the exchange modulation Rabi splitting requires that either the tip or the surface spins, or both, have to be misaligned with respect to the applied field.

\section{ELECTRIC CONTROL OF THE RESONANCE FREQUENCY}

In the EPR-STM experiments, there is a DC bias, with amplitude $V_{\mathrm{DC}}$, superimposed to the $\mathrm{AC}$ bias. In this section, we consider the shift of the resonance frequency of a $S=1 / 2$ adatom with anisotropic $g$ factor on account of the DC electric field between the tip and the surface. We consider the case of $\mathrm{Ti}-\mathrm{H} / \mathrm{MgO}$. The underlying mechanism is the same that gives rise to the spin transitions: Application of an off-plane electric field induces a strain $\delta z$ of the bond between the Ti adatom and the oxygen atom underneath [see Eq. (23)]. This leads to a modulation of the crystal field parameters $D$ and $F$ that in turn shifts the $g$ tensor.

In the case of the $g$-factor modulation, we can obtain an expression for the shift of the resonance frequency for a given DC modulation $\delta g_{x}$ and $\delta g_{z}$ of the $g$ tensor, up to linear order in $\Delta g_{a}$,

$$
\hbar \delta \omega \simeq \mu_{B}^{2} \frac{g_{x} \delta g_{x} B_{x}^{2}+g_{z} \delta g_{z} B_{z}^{2}}{\Delta_{z}},
$$

where $\Delta_{z}$ is the unperturbed modulation. We emphasize that $\delta g_{x}$ and $\delta g_{z}$ in Eq. (50) are the time-independent contributions 


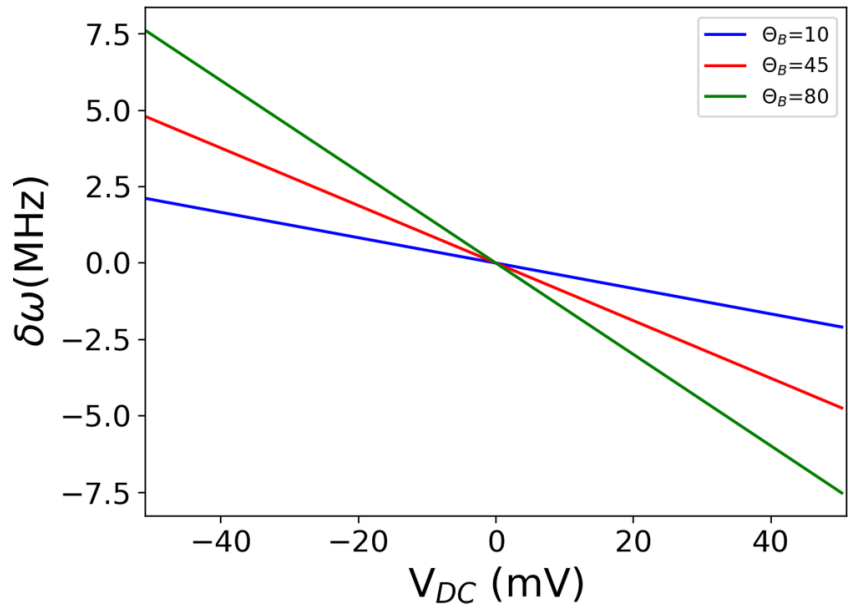

FIG. 8. Shift of the resonance frequency for $B=1 \mathrm{~T}, \lambda=$ $10 \mathrm{meV}, D=-255 \mathrm{meV}, F=1.4 \lambda \mathrm{meV}, k=290 \mathrm{eV} / \mathrm{nm}^{2}$, and $d=5 \AA$ for three different magnetic field angles.

to the $g$-factor anisotropy that arise from application of a DC electric field.

In Fig. 8, we plot the shift of the resonance frequency as a function of $V_{\mathrm{DC}}$ for a Ti-H adatom on $\mathrm{MgO}$. The calculation is carried out with the Hamiltonian Eq. (7). We take $D=$ $-255 \mathrm{meV}, F=1.4 \lambda=14.0 \mathrm{meV}$, and $B=1 \mathrm{~T}$. In order to compute $\delta z$, we take $k=290 \mathrm{eV} / \mathrm{nm}^{2}$ and a tip-MgO distance of $d=5 \AA$ [11]. The shift scales inversely with $d$. We consider three orientations for $\vec{B}$, forming angles $\theta_{B}=$ $10^{\circ}, 45^{\circ}, 80^{\circ}$. Expectedly, the resulting modulation is linear in $V_{\mathrm{DC}}$ and the effect is larger for fields off-plane, on account of the fact that $\Delta g_{z}>\Delta g_{x}$. With state of the art STM-EPR, the spectral resolution is around $3 \mathrm{MHz}$. Therefore, the shift might be observed for large values of $V_{\mathrm{DC}}$.

The exchange with the tip also contributes to the shift in the EPR frequency $[6,11,26]$. Unlike the $g$-factor modulation mechanism, the exchange contribution is tip dependent and decays exponentially with $d$. Therefore, for larger tip-surface distance $d$, the $g$-factor modulation should dominate. Electric shift of the spin resonance was observed experimentally in bulk $\mathrm{MgO}$ doped with $\mathrm{Cr}$ [41], by means of conventional EPR.

\section{DISCUSSION AND CONCLUSIONS}

The main idea of this paper is that electric fields can modulate the $g$-tensor anisotropy of magnetic adatoms, and that could be used to drive spin transitions. Our work comes motivated by recent EPR-STM experiments [3-12]. The electric modulation mechanism discussed here is an atomic scale version of the modulation of the $g$ factor of electrons $[19,42]$ and holes [43] in semiconductor nanostructures that lies at the heart of some well-known spintronics [44] and is becoming a resource in the manipulation of spin qubits $[45,46]$. At the atomic scale, the modulation proposed here occurs by controlling the weight of two orbital states with $\ell_{z}= \pm 2$ that have different orbital couplings.

We now briefly discuss some points of our work that could be improved. The derivation of the crystal field parameters for $\mathrm{Ti}-\mathrm{H} / \mathrm{MgO}$ could be improved using a Wannierization
[26,47]. In addition, we could also improve the model Eq. (7) by including the effects of hybridization between the $d$ orbitals of Ti and the $p$ and $s$ orbitals of oxygen and $s$ orbitals of titanium and hydrogen, as well as the effect of charge fluctuations [48].

Our estimation of the piezoelectric stretching could be improved in several ways. First, we are treating the silver substrate and most of the $\mathrm{MgO}$ as completely rigid. We have verified that keeping the $\mathrm{MgO}$ layer completely rigid, or letting a few atoms of the $\mathrm{MgO}$ layer close to the Ti adatom relax, has a minor impact on our estimate of $k$. We have also treated the potential as quasistatic. Whereas this is probably a good approximation, it the gigahertz frequency might resonate with long wavelengths off-plane $\mathrm{MgO}$ phonons that would definitely change the Ti-tip distance, very important for the exchange modulation mechanism $[11,26]$ and perhaps the Ti$\mathrm{O}$ distance as well.

The $g$ factor observed experimentally for $\mathrm{Ti}-\mathrm{H} / \mathrm{MgO}$ is $g \simeq 1.8$ for magnetic fields pointing almost in plane. From our theory, we expect a larger value, closer to $g_{x}=1.97$. There are two possible reasons for this discrepancy. First, the coupling to silver, ignored both in our DFT calculation and in the model, could distort slightly the electronic cloud of Ti-H, which would in turn change $D$ and $F$ and thereby $g_{x}$ and $g_{z}$. Second, and also related to silver, the Kondo coupling to the substrate electrons is expected to renormalize $g$, in analogy to the Knight shift $[49,50]$. In the case of an isotropic interaction, the renormalization of the $g$ factor reads, up to first order in the Kondo exchange between the adatom and the substrate electrons, $J_{s}$ :

$$
\delta \Delta_{z}=-\frac{\Delta_{z}}{2} g_{s} \rho J_{s}
$$

where $\rho_{s}$ is the density of states of substrate at the Fermi energy and $g_{s}$ is the $g$ factor of the substrate electrons. The sign of $J_{s}$ is positive for antiferromagnetic exchange, which is expected in this system, since Kondo effect was observed for $\mathrm{Cu} / \mathrm{MgO}[10]$. Therefore, the Kondo interaction could reduce the $g$ factor of Ti-H.

We now summarize the main results of this work:

(1) We have shown that an anisotropic time-dependent modulation of the $g$ tensor induces electronic spin transitions, described by Hamiltonian Eq. (5) and characterized by a Rabi coupling given in Eq. (6).

(2) We have worked out an analytical theory for the anisotropic $g$ factor of the Ti-H $S=1 / 2$ adatom on $\mathrm{MgO}$ and we have benchmarked it against DFT calculations. Our theory relates $g$ with crystal field parameters $D$ and $F$, as well as Ti spin orbit coupling $\lambda$ [Eqs. (15) and (22) and Fig. 4].

(3) We have computed the modulation of the $g$-tensor anisotropy due to the piezoelectric strain of the Ti-H chemisorbed on an oxygen atom on $\mathrm{MgO}$ and we have estimated the resulting Rabi coupling (see Fig. 6). We have found that is much smaller than the one observed experimentally, confirming the dominance of the exchange modulation mechanism $[11,26]$. However, we have shown that for heavier adatoms with much larger spin orbit coupling $\lambda$, this mechanism could be efficient.

(4) We have studied to what extent the crystal field mechanism for EPR-STM, proposed by Baumann et al. to 
understand the experiments for $\mathrm{Fe}$ on $\mathrm{MgO}$ [3], could be ascribed to the modulation of the $g$ factor. We find that the dominant contributions to the crystal field mechanism come from the modulation of the zero field splitting parameters.

(5) We have also proposed that a DC voltage can shift the $g$ factor and thereby the Zeeman splitting, and we have computed the effect for the case of Ti-H [see Eq. (50) and Fig. 8]. This shift provides an additional knob to fine-tune the resonance frequency.

(6) We have discussed the impact on the hyperfine coupling of the $g$-factor anisotropy and its electric modulation [see Eq. (34)].

(7) We have discussed the role of the $g$-factor anisotropy of $\mathrm{Ti}-\mathrm{H} / \mathrm{MgO}$ to enable the exchange-modulation mechanism for EPR-STM [Eq. (48)]. In the absence of adatom $g$-factor anisotropy, the exchange-modulation EPR-STM mechanism can only work if the tip moment is not aligned with the applied field [see Eq. (49)].

In this work, we have focused mostly on $\mathrm{Ti}-\mathrm{H} / \mathrm{MgO}$ and $\mathrm{Fe} / \mathrm{MgO}$, although most of the ideas can be applied or extended to the case of other atoms. Most notably, the case of $\mathrm{Cu} / \mathrm{MgO}$ will be the subject of a future publication.

\section{ACKNOWLEDGMENTS}

We acknowledge Kai Yang, for fruitful discussions. J.F.-R. acknowledges financial support from FCT Grants No. P2020-PTDC/FIS-NAN/4662/2014 and No. UTAPEXPL/NTec/0046/2017, as well as Generalitat Valenciana funding Prometeo2017/139 and MINECO-Spain (Grant No. MAT2016-78625-C2). A.F. acknowledges hospitality from the Department of Physics of Univeridad de Alicante. A.F., S.A.R., and S.S.G. acknowledge financial support from CONICET (No. PIP11220150100327 and No. PUE22920170100089CO).

\section{APPENDIX A: CALCULATION OF RABI CONSTANT FOR SPIN MODEL}

In this Appendix, we compute the Rabi coupling defined in Eq. (5). We consider the general situation for a $S=1 / 2$ :

$$
\mathcal{H}=\vec{b}_{0} \cdot \vec{S}+\vec{b}_{1}(t) \cdot \vec{S}
$$

where

$$
\vec{b}_{0}=\left|\vec{b}_{0}\right|\left(\sin \theta_{0}, 0, \cos \theta_{0}\right)
$$

and

$$
\vec{b}_{1}(t)=\left|\vec{b}_{1}\right| \cos (2 \pi f t)\left(\sin \theta_{1}, 0, \cos \theta_{1}\right) .
$$

We shall give explicit expressions for $\vec{b}_{0}$ and $\vec{b}_{1}$ below, where we consider independently the exchange modulation and the $g$-factor modulation mechanism.

The eigenstates of $H_{=} \mu_{B} \vec{b}_{0} \cdot \vec{S} \quad$ satisfy $\quad H_{0}| \pm\rangle=$ $\pm \mu_{B}\left|\vec{b}_{0}\right|| \pm\rangle$ and are given by

$$
\begin{aligned}
& |+\rangle=\cos \frac{\theta_{0}}{2}|\uparrow\rangle+\sin \frac{\theta_{0}}{2}|\downarrow\rangle, \\
& |-\rangle=\sin \frac{\theta_{0}}{2}|\uparrow\rangle-\cos \frac{\theta_{0}}{2}|\downarrow\rangle .
\end{aligned}
$$

We obtain the matrix element of the spin operators in the basis of eigenstates:

$$
\begin{gathered}
\left\langle+\left|S_{x}\right|-\right\rangle=\frac{1}{2}\left(\sin ^{2} \frac{\theta_{0}}{2}-\cos ^{2} \frac{\theta_{0}}{2}\right)=-\frac{1}{2} \cos \theta_{0}, \\
\left\langle+\left|S_{z}\right|-\right\rangle=\sin \frac{\theta_{0}}{2} \cos \frac{\theta_{0}}{2}=\frac{1}{2} \sin \theta_{0} .
\end{gathered}
$$

We can now write the general expression

$$
\Omega=\frac{\left|\vec{b}_{1}\right|}{2}\left(-\sin \theta_{1} \cos \theta_{0}+\cos \theta_{1} \sin \theta_{0}\right) .
$$

This can be further simplified to

$$
\Omega=\frac{\left|\vec{b}_{1}\right|}{2} \sin \left(\theta_{1}-\theta_{0}\right)=\frac{1}{2} \frac{\vec{b}_{0} \cdot \vec{b}_{1}}{\left|\vec{b}_{0}\right|} .
$$

\section{Expression for $\boldsymbol{\Omega}$ for the $\boldsymbol{g}$-factor modulation}

We now apply Eq. (A9) for the case of the $g$-factor modulation. We now write up

$$
\vec{b}_{0}=\mu_{B}\left(g_{x} B_{x}, 0, g_{z} B_{z}\right)
$$

and

$$
\vec{b}_{1}=\mu_{B}\left(\delta g_{x} B_{x}, 0, \delta g_{z} B_{z}\right) .
$$

Explicitly, we write

$$
\sin \theta_{0}=\frac{g_{x} B_{x}}{\sqrt{\left(g_{x} B_{x}\right)^{2}+\left(g_{z} B_{z}\right)^{2}}}
$$

and

$$
\cos \theta_{0}=\frac{g_{z} B_{z}}{\sqrt{\left(g_{x} B_{x}\right)^{2}+\left(g_{z} B_{z}\right)^{2}}} .
$$

We thus write

$$
\Omega=\frac{\mu_{B}}{2}\left(-\delta g_{x} B_{x} \cos \theta+\delta g_{z} B_{z} \sin \theta\right) .
$$

We now use Eqs. (A12) and (A13) to express $B_{x}$ and $B_{y}$ in terms of $\theta, g_{x}, g_{z}, B_{x}, B_{z}$ and we obtain

$$
\Omega=\frac{\mu_{B}}{2}\left|\vec{b}_{0}\right| \cos \theta_{0} \sin \theta_{0}\left(-\frac{\delta g_{x}}{g_{x}}+\frac{\delta g_{z}}{g_{z}}\right) .
$$

Now we use $\cos \theta_{0} \sin \theta_{0}=\frac{1}{2} \sin 2 \theta_{0}$ to obtain

$$
\Omega=\frac{\mu_{B}}{4}\left|\vec{b}_{0}\right| \sin 2 \theta_{0}\left(\frac{\delta g_{z}}{g_{z}}-\frac{\delta g_{x}}{g_{x}}\right) .
$$

We now write the Zeeman splitting

$$
\Delta_{Z}=\mu_{B}\left|\vec{b}_{0}\right|,
$$

so that we obtain the expression

$$
\Omega=\frac{\Delta_{Z}}{4} \sin 2 \theta\left(\frac{\delta g_{z}}{g_{z}}-\frac{\delta g_{x}}{g_{x}}\right) .
$$

\section{Expression for $\boldsymbol{\Omega}$ for the exchange modulation}

We now consider a Hamiltonian for a $S=1 / 2$ surface spin where, in addition to the Zeeman interaction, there is a exchange coupling to the magnetic moment of the tip:

$$
\mathcal{V}_{\text {exch }}=J(z) \vec{n}_{T} \cdot \vec{S},
$$


where

$$
\vec{n}_{T}=\left[\cos \left(\theta_{B}+\delta\right), 0, \sin \left(\theta_{B}+\delta\right)\right]
$$

and $\vec{B}=B_{0}\left(\cos \left(\theta_{B}\right), 0, \sin \left(\theta_{B}\right)\right)$.

We define the spin splitting

$$
\Delta_{Z}^{*} \equiv \sqrt{\left(g_{x} \mu_{B} B_{x}+J n_{x}\right)^{2}+\left(g_{z} \mu_{B} B_{z}+J n_{z}\right)^{2}}
$$

and we express the angles as

$$
\sin \theta_{0}=\frac{g_{x} B_{x}+J n_{x}}{\Delta_{Z}^{*}}
$$

and

$$
\cos \theta_{0}=\frac{g_{z} B_{z}+J n_{z}}{\Delta_{Z}^{*}} .
$$

For the time-dependent component, we now ignore the modulation of the $g$ factors and we only consider the modulation of the exchange that we write up as $\delta J \cos 2 \pi f t$. We thus can write

$$
\vec{b}_{1}(t)=\delta J\left(n_{x}, 0, n_{z}\right) .
$$

After some algebra, we obtain

$$
\Omega_{J}=\frac{\delta J}{2 \Delta_{z}^{*}}\left(g_{x} \mu_{B} \cdot B_{x} n_{z}-g_{z} \mu_{B} B_{z} n_{x}\right) .
$$

Since we are interested in the role of the $g$-factor anisotropy, we make it explicit and we write

$$
\begin{gathered}
g_{x}=g+\Delta g_{x}, \\
g_{z}=g+\Delta g_{z},
\end{gathered}
$$

where $\Delta g_{x}$ and $\Delta g_{z}$ are the static contributions to the $g$-factor anisotropy. We now define

$$
\mathcal{E}_{J} \equiv \frac{\delta J}{2 \Delta_{z}^{*}} g \mu_{B} B
$$

so that the expression for the Rabi reads

$$
\begin{aligned}
\Omega_{J}= & \mathcal{E}_{J}\left(\cos \theta_{B} \sin \left(\theta_{B}+\delta\right)-\sin \theta_{B} \cos \left(\theta_{B}+\delta\right)\right) \\
& +\mathcal{E}_{J}\left(\frac{\Delta g_{x}}{g} \cos \theta_{B} \sin \left(\theta_{B}+\delta\right)-\frac{\Delta g_{z}}{g} \sin \theta_{B} \cos \left(\theta_{B}+\delta\right)\right) .
\end{aligned}
$$

We can now write this up as

$$
\begin{aligned}
\Omega_{J}= & \mathcal{E}_{J} \sin \delta \\
& +\mathcal{E}_{J}\left(\frac{\Delta g_{x}}{g} \cos \theta_{B} \sin \left(\theta_{B}+\delta\right)-\frac{\Delta g_{z}}{g} \sin \theta_{B} \cos \left(\theta_{B}+\delta\right)\right) .
\end{aligned}
$$

[1] Y. Manassen, R. J. Hamers, J. E. Demuth, and A. J. Castellano, Jr., Phys. Rev. Lett. 62, 2531 (1989).

[2] A. V. Balatsky, M. Nishijima, and Y. Manassen, Adv. Phys. 61, 117 (2012).

[3] S. Baumann, W. Paul, T. Choi, C. P. Lutz, A. Ardavan, and A. J. Heinrich, Science 350, 417 (2015).

[4] F. D. Natterer, K. Yang, W. Paul, P. Willke, T. Choi, T. Greber, A. J. Heinrich, and C. P. Lutz, Nature (London) 543, 226 (2017).
After some algebra, we obtain

$$
\begin{aligned}
\Omega_{J}= & \mathcal{E}_{J} \sin \delta+\mathcal{E}_{J}\left(\frac{\Delta g_{x}}{g}-\frac{\Delta g_{z}}{g}\right) \cos \theta_{B} \sin \theta_{B} \cos \delta \\
& +\mathcal{E}_{J}\left(\frac{\Delta g_{x}}{g} \cos ^{2} \theta_{B}+\frac{\Delta g_{z}}{g} \sin ^{2} \theta_{B}\right) \sin \delta .
\end{aligned}
$$

\section{APPENDIX B: ESTIMATION OF SOC FROM NIST DATABASE}

From the NIST database [51], we obtain the experimental values for Ti(IV) with an outermost electronic configuradion $d^{1}$. The lowest energy levels have $L=2$ and $S=1 / 2$, with $J=3 / 2$ and $J=5 / 2$. Their energy splitting is $\Delta E=$ $47.3 \mathrm{meV}$. We can relate this to the spin orbit coupling using $J=L+S$ and

$$
\lambda \vec{L} \cdot \vec{S}=\frac{\lambda}{2}[J(J+1)-L(L+1)-S(S+1)] .
$$

From here, we obtain

$$
E(J)=\frac{\lambda}{2}[J(J+1)-L(L+1)-S(S+1)]
$$

and

$E(J+1)-E(J)=\frac{\lambda}{2}[J+1(J+2-J)]=(J+1) \lambda$.

Since $J+1=\frac{5}{2}$, we obtain $\lambda_{\mathrm{IV}}=\frac{2}{5} \Delta E_{\mathrm{IV}}=18.9 \mathrm{meV}$. If we consider Ti(III), we have $J+1=3$ and $\Delta E_{\mathrm{III}}=22.9 \mathrm{meV}$. This yields $\lambda_{\mathrm{IV}}=\frac{1}{3} \Delta E_{\mathrm{III}}=7.63$.

It is apparent that the strength of the atomic spin orbit coupling depends on the charge imbalance in the Ti. One can expect that value of $\lambda$ for $\mathrm{Ti}-\mathrm{H}$ on $\mathrm{MgO}$ must be in between these two values.

\section{APPENDIX C: RELATION BETWEEN THE $\left(\ell^{+}\right)^{4}+\left(\ell^{-}\right)^{4}$ AND THE $\ell_{x}^{4}+\ell_{y}^{4}$ TERMS}

In this Appendix, we discuss the connection between these two crystal field operators that we have used for $\mathrm{Ti}$ and $\mathrm{Fe}$. The choice is a matter of convenience. After some algebra, the relation between these two operators is

$$
\ell_{x}^{4}+\ell_{y}^{4}=\frac{\left(\ell^{+}\right)^{4}+\left(\ell^{-}\right)^{4}}{8}+24 I-\frac{91}{12} \ell_{z}^{2}+\frac{7}{12} \ell_{z}^{4} .
$$

Thus, it is apparent that the last two terms can can be reabsorbed as a renormalization of the $D \ell_{z}^{2}$ term, plus a shift of the $\ell_{z}=0$ level, that plays a very minor role in the discussion for $\mathrm{Ti}-\mathrm{H} / \mathrm{MgO}$. 
[8] P. Willke, Y. Bae, K. Yang, J. L. Lado, A. Ferrón, T. Choi, A. Ardavan, J. Fernández-Rossier, A. J. Heinrich, and C. P. Lutz, Science 362, 336 (2018).

[9] Y. Bae, K. Yang, P. Willke, T. Choi, A. J. Heinrich, and C. P. Lutz, Sci. Adv. 4, eaau4159 (2018).

[10] K. Yang, P. Willke, Y. Bae, A. Ferrón, J. L. Lado, A. Ardavan, J. Fernández-Rossier, A. J. Heinrich, and C. P. Lutz, Nat. Nanotech. 13, 1120 (2018).

[11] K. Yang, W. Paul, F. D. Natterer, J. L. Lado, Y. Bae, P. Willke, T. Choi, A. Ferrón, J. Fernández-Rossier, A. J. Heinrich et al., Phys. Rev. Lett. 122, 227203 (2019).

[12] P. Willke, K. Yang, Y. Bae, A. J. Heinrich, and C. P. Lutz, Nat. Phys. 15, 1005 (2019).

[13] P. Willke, A. Singha, X. Zhang, T. Esat, C. Lutz, A. J. Heinrich, and T. Choi, Nano Lett. 19, 8201 (2019).

[14] F. D. Natterer, F. Patthey, T. Bilgeri, P. R. Forrester, N. Weiss, and H. Brune, Rev. Sci. Instrum. 90, 013706 (2019).

[15] T. Seifert, S. Kovarik, C. Nistor, L. Persichetti, S. Stepanow, and P. Gambardella, arXiv:1908.03379 (unpublished).

[16] S. Thiele, F. Balestro, R. Ballou, S. Klyatskaya, M. Ruben, and W. Wernsdorfer, Science 344, 1135 (2014).

[17] C. Godfrin, A. Ferhat, R. Ballou, S. Klyatskaya, M. Ruben, W. Wernsdorfer, and F. Balestro, Phys. Rev. Lett. 119, 187702 (2017).

[18] E. I. Rashba, Sov. Phys. Solid State 2, 1109 (1960).

[19] Y. Kato, R. Myers, D. Driscoll, A. Gossard, J. Levy, and D. Awschalom, Science 299, 1201 (2003).

[20] Y. Tokura, W. G. van der Wiel, T. Obata, and S. Tarucha, Phys. Rev. Lett. 96, 047202 (2006).

[21] M. Pioro-Ladriere, T. Obata, Y. Tokura, Y.-S. Shin, T. Kubo, K. Yoshida, T. Taniyama, and S. Tarucha, Nat. Phys. 4, 776 (2008).

[22] R. L. Bell, Phys. Rev. Lett. 9, 52 (1962).

[23] R. E. George, J. P. Edwards, and A. Ardavan, Phys. Rev. Lett. 110, 027601 (2013).

[24] B. M. Hoffman, J. Phys. Chem. 98, 11657 (1994).

[25] P. Berggren and J. Fransson, Sci. Rep. 6, 25584 (2016).

[26] J. L. Lado, A. Ferrón, and J. Fernández-Rossier, Phys. Rev. B 96, 205420 (2017).

[27] A. M. Shakirov, A. N. Rubtsov, and P. Ribeiro, Phys. Rev. B 99, 054434 (2019).

[28] J. Reina Galvez, C. Wolf, F. Delgado, and N. Lorente, Phys. Rev. B 100, 035411 (2019).

[29] Given the $C_{4}$ symmetry of the adatom on the oxygen position in $\mathrm{MgO}, x$ and $y$ directions are equivalent and we assume $B_{y}=0$.
[30] P. Giannozzi, S. Baroni, N. Bonini, M. Calandra, R. Car, C. Cavazzoni, D. Ceresoli, G. L. Chiarotti, M. Cococcioni, I. Dabo et al., J. Phys.: Condens. Matter 21, 395502 (2009).

[31] J. P. Perdew, K. Burke, and M. Ernzerhof, Phys. Rev. Lett. 80, 891 (1998).

[32] P. E. Blöchl, Phys. Rev. B 50, 17953 (1994).

[33] C. J. Pickard and F. Mauri, Phys. Rev. Lett. 88, 086403 (2002).

[34] S. Baumann, F. Donati, S. Stepanow, S. Rusponi, W. Paul, S. Gangopadhyay, I. G. Rau, G. E. Pacchioni, L. Gragnaniello, M. Pivetta et al., Phys. Rev. Lett. 115, 237202 (2015).

[35] M. J. Klein, Am. J. Phys. 20, 65 (1952).

[36] A. Garg, Europhys. Lett. 22, 205 (1993).

[37] W. Wernsdorfer and R. Sessoli, Science 284, 133 (1999).

[38] F. Delgado, S. Loth, M. Zielinski, and J. Fernández-Rossier, Europhys. Lett. 109, 57001 (2015).

[39] R. E. George, W. Witzel, H. Riemann, N. V. Abrosimov, N. Nötzel, M. L. W. Thewalt, and J. J. L. Morton, Phys. Rev. Lett. 105, 067601 (2010).

[40] M. Shafiei, K. C. Nowack, C. Reichl, W. Wegscheider, and L. M. K. Vandersypen, Phys. Rev. Lett. 110, 107601 (2013).

[41] E. B. Royce and N. Bloembergen, Phys. Rev. 131, 1912 (1963).

[42] H. Malissa, W. Jantsch, M. Mühlberger, F. Schäffler, Z. Wilamowski, M. Draxler, and P. Bauer, Appl. Phys. Lett. 85, 1739 (2004).

[43] J. H. Prechtel, F. Maier, J. Houel, A. V. Kuhlmann, A. Ludwig, A. D. Wieck, D. Loss, and R. J. Warburton, Phys. Rev. B 91, 165304 (2015).

[44] S. Datta and B. Das, Appl. Phys. Lett. 56, 665 (1990).

[45] E. Kawakami, P. Scarlino, D. R. Ward, F. Braakman, D. Savage, M. Lagally, M. Friesen, S. N. Coppersmith, M. A. Eriksson, and L. Vandersypen, Nat. Nanotech. 9, 666 (2014).

[46] A. Laucht, J. T. Muhonen, F. A. Mohiyaddin, R. Kalra, J. P. Dehollain, S. Freer, F. E. Hudson, M. Veldhorst, R. Rahman, G. Klimeck et al., Sci. Adv. 1, e1500022 (2015).

[47] A. Ferrón, F. Delgado, and J. Fernández-Rossier, New J. Phys. 17, 033020 (2015).

[48] A. Ferrón, J. L. Lado, and J. Fernández-Rossier, Phys. Rev. B 92, 174407 (2015)

[49] D. C. Langreth and J. W. Wilkins, Phys. Rev. B 6, 3189 (1972).

[50] F. Delgado, C. Hirjibehedin, and J. Fernández-Rossier, Surf. Sci. 630, 337 (2014).

[51] D. E. Kelleher, W. C. Martin, W. L. Wiese, J. Sugar, J. R. Fuhr, K. Olsen, A. Musgrove, P. J. Mohr, J. Reader, and G. Dalton, Phys. Scr. T83, 158 (1999). 